\title{
Intraepidermal Nerve fiber Density in healthy Subjects with Diabetic Family History. Is Neuropathy an early Biomarker? A Pilot study
}

Moises Rubio-Hernandez ${ }^{1}$, Ildefonso Rodriguez-Leyva ${ }^{1 *}$, Juan Pablo Castanedo-Cazares ${ }^{2}$, Olga Johnson-Ponce ${ }^{3}$, Ma del Pilar FonsecaLeal $^{1}$, Cornelia Fuentes-Ahumada ${ }^{2}$, Edgar Abad-Guangorena ${ }^{2}$, Valentin Aaron Jerez-Valdez ${ }^{4}$

${ }^{1}$ Neurology Department, Hospital Central Ignacio Morones Prieto, San Luis Potosí, Mexico.

${ }^{2}$ Dermatology Department, Hospital Central Ignacio Morones Prieto, San Luis Potosí, Mexico.

${ }^{3}$ Pathology Department, Hospital Central Ignacio Morones Prieto, San Luis Potosí, Mexico.

${ }^{4}$ Universidad Autónoma de Baja California, Mexico.

*Corresponding Author: Ildefonso Rodriguez-Leyva, Neurology Department, Hospital Central Ignacio Morones Prieto, San Luis Potosí, Mexico.

Received date: March 06, 2021; Accepted date: March 22, 2021; Published date: March 29, 2021

Citation: Rubio-Hernandez M., Rodriguez-Leyva I, Castanedo-Cazares JP, Johnson-Ponce O., Fonseca-Leal MDP., et al. (2021) Intraepidermal Nerve fiber Density in healthy Subjects with Diabetic Family History. ¿Is Neuropathy an early Biomarker? A Pilot study. J. Neuroscience and Neurological Surgery. 8(3); DOI:10.31579/2578-8868/169

Copy rights: @ 2021 Ildefonso Rodriguez Leyva, This is an open-access article distributed under the terms of The Creative Commons Attribution License, which permits unrestricted use, distribution, and reproduction in any medium, provided the original author and source are credited

\section{Abstract}

Objective: Evaluate the intraepidermal nerve fiber density in healthy subjects with diabetic family history compared with diabetic patients and controls.

Introduction: Neuropathy is the most prevalent chronic complication of diabetes, presenting various symptoms that interfere with daily living activities, psychosocially disability, and reducing life quality. The skin biopsy is recognized as a minimally invasive procedure that allows morphometric quantification of intraepidermal nerve fibers and has made possible the study of peripheral neuropathies involving thin fibers that traditional methods cannot diagnose.

Methods: Analytical cross-sectional observational pilot study with seven patients per group including healthy, diabetic, and healthy with diabetic family history subjects. For the statistical analysis, we used the R package, R software version 3.3.2, with a confidence level of $95 \%$. The research was performed with ANOVA and KruskalWallis test to test the primary objective.

Results: The density of intraepidermal nerve fibers is similar between the group with diabetic family history $6.8 \pm$ $2.1(3.5-10.1)$ and diabetic patients $6.3 \pm 2.9$ (3.5 - 7.05) while the control group reported a density in parameters of normality of $10 \pm 1.2(8.2-10.1)$ with a $p=0.01$ between the three groups. The decrease of intraepidermal nerve fibers showed a tendency to decrease with increasing age and BMI with a ratio coefficient for age of $\mathrm{r}=-0.342,95 \%$ CI $(-0.67-0.106), p=0.129$; and for BMI of $r=-0.36,95 \%$ CI $(-0.685-0.0847), p=0.109$.

Conclusion: Intraepidermal nerve fiber density is decreased in subjects with a family history of diabetes mellitus type 2 and even more so in diabetics, with no statistical difference.

Keywords: diabetic neuropathy; symmetrical distal polyneuropathy; diabetes mellitus type 1 (dm1); diabetes mellitus type $2(\mathrm{dm} 2)$; intradermal nerve fibers; intradermal nerve fiber density; glycosylated hemoglobin

Diabetic neuropathy (DN) is the most prevalent and painful chronic complication of diabetes [1].

Diabetic distal symmetric sensorimotor polyneuropathy (DSSP) is the most common clinical subtype, which accounts for approximately $75 \%$ of cases. Observational cohorts suggest that DSPN appears in at least 20\% of people with DM1 when they have 20 years of disease progression and is detected in at least $10-15 \%$ of newly diagnosed patients with DM2, increasing in frequency to $50 \%$ after ten years of disease duration [1-3].

Symptoms are predominantly sensitive length-dependent, i.e., they affect the feet first and present proximal progression. They can be divided into 
"positive" (tingling, burning, shooting pain, and other abnormal sensations) or "negative" (sensory loss, weakness, and numbness). Motor symptoms are less common and occur late.

Painful diabetic neuropathy occurs in $10-26 \%$ of patients, mainly due to thin fibers' involvement. There is an exaggerated response to painful and inclusive, not usual painful stimuli (hyperalgesia), and pain evoked by contact with, for example, socks, shoes, or bed sheets (allodynia) worsens at night. Neuropathic pain interferes with daily living activities, is psychosocially disabling, and reduces the quality of life. On the contrary, when it affects thick fibers, patients present numbness, tingling without pain, and loss of sensation of protection. They report feelings such as "having their feet trapped in concrete" or "walking on thick shoes," the loss of pain sensation allows the patient to walk on the neuropathic ulcers, promoting chronicity that is frequently associated with infection [1-3].

All sensory modalities may be affected, reduced vibration detected with $128 \mathrm{~Hz}$ tuning fork is an early indicator of neuropathy, 1.0-g. SemmesWeinstein monofilament can be used to detect changes in sensation. In contrast, abnormal sense with 10-g. Monofilament, including assessing the dorsal aspect of the first orthotic of both feet described by Perkins et al., indicates an increased risk of ulceration and amputation [3].

Treatment involves pharmacological and non-pharmacological measures such as lifestyle interventions through diet and exercise that can prevent or reverse polyneuropathy. However, the main recommendation is prevention through strict glycemic control. Therapeutic options for symptomatic management of ND pain include gabapentin, pregabalin, topiramate, and venlafaxine [1,2].

DSSP can affect different nerve fibers and various degrees; this implies difficulties when selecting the screening instrument in the population; this is mainly due to the subjective interpretation that patients have of the sensory stimuli commonly applied, which results in poor reproducibility.

Skin biopsy is a minimally invasive procedure that allows morphometric quantification of intraepidermal nerve fibers (IENF), expressed by section length (IENF/mm). This ratio has been widely used in recent years for the study of peripheral neuropathies involving thin nerve fibers that are not diagnosed by physical $(128 \mathrm{~Hz}$ tuning fork vibration test, SemmesWeinstein monofilament test) neurophysiological (nerve conduction studies), or traditional neuropathological (sural nerve biopsy) tests [4-6].

Skin samples can be taken from anybody's site. However, the most common areas are the distal portion of the leg $(10 \mathrm{~cm}$ above the lateral malleolus) and the thigh (20 cm below the iliac spine). The preferred technique is a $3-\mathrm{mm}$ punch biopsy, as it is minimally invasive and safe under local anesthesia. Quantification of linear INFD density is performed on at least three $50 \mu \mathrm{m}$ thick sections per biopsy, fixed in $2 \%$ periodate-lysine-formaldehyde(PLP) for brightfield microscopy, or Zamboni fixative solution (2\% picric acid-formaldehyde) for confocal microscopy, by immunohistochemistry using rabbit polyclonal anti-PGP 9.5.5-8 antibodies.

PGP 9.5 is a carboxyl-terminal ubiquitin hydrolase found mainly in neurons and accompanies the slow axonal transport component. It is widely distributed in the peripheral nervous system and is a non-diffuse pan-axonal marker [5-7].

Brightfield immunohistochemistry and indirect immunofluorescence with confocal microscopy are the two most used immunostaining methods for investigating cutaneous innervation. Based on the results obtained from various studies, typical values for the density of INFD in the distal portion of healthy subjects using brightfield immunohistochemistry are currently available; these values range from $12.4+4.6$ fibers $/ \mathrm{mm}$ to $15+$ 5/mm.3,4 Furthermore, it has been observed that the density of INFD shows a decreasing gradient from proximal to distal regions of the body, being $60 \%$ higher in the thigh region than in the supra malleolar area $[8,9]$.

In patients with ND, low INFD density was first described by Levy et al [10,11], and confirmed by Kennedy et al. [9]. Epidermal denervation must increase with the duration of diabetes, and even nerve fiber reduction has been observed in subjects with prediabetes [7,8] In 2004, Pittenger et al. [8] described decreased mean dendritic length and INFD density in diabetic and non-diabetic patients with neuropathy compared to a healthy control group. The degree of decrease in INFD density was greater in patients with a longer duration of diabetes.

The European Federation of Neurological Societies/Peripheral Nerve Society made a review and update of the guidelines for the use of skin biopsies for the diagnosis of peripheral neuropathy were made in 2010 [12]. These guidelines indicate reference values for INFD density in healthy subjects using the brightfield immunohistochemistry technique; however, not for confocal immunofluorescence. Also, serial skin biopsies could help detect early INFD density changes, which could predict neuropathy progression and assess INFD degeneration and regeneration rates. However, studies are needed to confirm the usefulness of this technique.

Reference values in healthy subjects from the 2010 guidelines range from $9.8+3.6$ to $13.8+6.7$ fibers $/ \mathrm{mm}$ [12]. The largest normative study included 188 healthy subjects stratified by age and gender, establishing values for each decade. Authors observed that the density of INFD in the distal portion of the leg is lower in men than in women and that weight and height do not have a significant impact, but that their values decrease with age.

After the 2010 guidelines, another study was conducted with 550 healthy participants. Patients with symmetric distal polyneuropathy had lower INFD density and dermal and epidermal nerve fiber length with higher axonal swelling rates than healthy controls $[13,14]$. Regarding the use of INFD as a biomarker of progression in diabetic neuropathy, Narayanaswamy et al [15] observed an average annual rate in INFD loss in patients with ND of 3.76 fibers $/ \mathrm{mm}$.

In 2016, Divisova et al [16] observed that the annual rate of INFD loss in patients with DM2 is several times higher than in healthy. Importantly, this study identified that the change in INFD density is not linear and should be expressed as a proportion of the initial INFD density to serve as a marker of the course of ND. The loss of INFD in these patients was less than that described by Narayanaswamy et al in 2012 [15]; however, patients in this first study had a longer duration of diabetes and worse glycemic control, supporting the theory that these factors accelerate the loss of thin fibers.

\section{Methods}

To fulfill the primary objective, we made an observational, crosssectional, analytical study was designed. The study's universe was formed by the patients who attended the outpatient services of the endocrinology, dermatology, and neurology departments of the Hospital Central "Dr. Ignacio Morones Prieto." Since there were no similar studies in the literature, a pilot study was carried out, including 21 patients, seven subjects per group [17]. Patients were formed according to the selection criteria, aged 18 to 65 years and of either sex. In group 1, healthy subjects with diabetic family history of first-degree diabetes mellitus type 2 with 
glycosylated hemoglobin levels of less than $5.7 \%$; in group 2, healthy subjects with no family history of diabetes mellitus type 2 and with glycosylated hemoglobin levels of less than 5.7\%; and in group 3, subjects with a diagnosis of type 2 diabetes mellitus with glycosylated hemoglobin levels of more than $6.5 \%$. All agreed to participate and signed a letter of informed consent.

All the patients who had a history of HIV, immune-mediated neuropathies such as systemic lupus erythematosus, Sjögren's syndrome, celiac disease, or sarcoidosis were excluded. Also, hereditary neuropathies such as Fabry disease, Charcot-Marie-Tooth, or Friedreich's ataxia. Besides leprosy, hypothyroidism, use of neurotoxic drugs, Parkinson's disease. We also excluded subjects with non-diabetic neuropathy.

Biopsy technique, tissue handling, and cutting procedures are described in Annex 1. The morphometric evaluation and the quantification of intraepidermal nerve fibers were performed by a certified pathologist who evaluated the slides in a blinded manner. Table 1 describes the variables studied.

\begin{tabular}{|l|l|l|l|l|}
\hline Variable & Operational definition & Possible values & Units & Type \\
\hline $\begin{array}{l}\text { Intraepidermal nerve } \\
\text { fibers }\end{array}$ & $\begin{array}{l}\text { Intraepidermal nerve fiber } \\
\text { density }\end{array}$ & $0-20$ & INFD/mm & Continued \\
\hline Study groups & $\begin{array}{l}\text { Risk factor for diabetic } \\
\text { neuropathy }\end{array}$ & Group 1 & N/A & Categorical \\
\hline Age & Patient's age attained & Group 2 & Years & Continuous \\
\hline Sex & The biological sex of the patient & Group 3 & N/A & Dichotomous \\
\hline HbA1c & $\begin{array}{l}\text { Metabolic control of DM2 in the } \\
\text { last three months }\end{array}$ & $18-65$ & $\%$ & Continued \\
\hline Height & $\begin{array}{l}\text { Height in centimeters of the } \\
\text { subject }\end{array}$ & $0=$ female & cm & Continued \\
\hline
\end{tabular}

Table 1: Variables included in the study.

\section{Statistical Analysis}

For the statistical analysis, we used the $\mathrm{R}$ package of the $\mathrm{R}$ software version 3.3.2 [18] with a confidence level of $95 \%$. The normality of the distribution of continuous variables was evaluated with the Shapiro Wilk test. Continuous variables are reported as mean (median) [Q1, Q3] (min-

max), discrete variables as frequencies (\%). The analysis was performed with ANOVA and Kruskal-Wallis test to test the primary objective.

\section{Results}

We included twenty-one subjects, 7 in each group, age, sex, weight, and height are described in Table 2.

\begin{tabular}{|l|l|l|l|l|}
\hline & $\begin{array}{l}\text { Total } \\
\mathbf{n = 2 1}\end{array}$ & $\begin{array}{l}\text { Group 1 } \\
\text { (Control) } \\
\mathbf{n = 7}\end{array}$ & $\begin{array}{l}\text { Group 2 } \\
\text { (HFA) } \\
\mathbf{n = 7}\end{array}$ & $\begin{array}{l}\text { Group 3 } \\
\text { DM2 } \\
\mathbf{n}=\mathbf{7}\end{array}$ \\
\hline Age (years) & $\begin{array}{l}39.4 \pm 12.1 \\
(18-59)\end{array}$ & $\begin{array}{l}36.8 \pm 13.9 \\
(20-59)\end{array}$ & $\begin{array}{l}38.5 \pm 11.4 \\
(25-54)\end{array}$ & $\begin{array}{l}42.7 \pm 11.9 \\
(18-54)\end{array}$ \\
\hline $\begin{array}{l}\text { Gender } \\
\text { Female } \\
\text { Male }\end{array}$ & $\begin{array}{l}13(62) \\
8(38)\end{array}$ & $\begin{array}{l}3(43) \\
4(57)\end{array}$ & $\begin{array}{l}6(86) \\
1(14)\end{array}$ & $\begin{array}{l}4(57) \\
3(43)\end{array}$ \\
\hline Weight (kg) & $\begin{array}{l}74.1 \pm 14.8 \\
(46.9-119)\end{array}$ & $\begin{array}{l}74.1 \pm 8.3 \\
(64-85)\end{array}$ & $\begin{array}{l}68.3 \pm 13.1 \\
(47-86)\end{array}$ & $\begin{array}{l}79.9 \pm 20.3 \\
(61.5-119)\end{array}$ \\
\hline Height (m) & $\begin{array}{l}1.64 \pm 0.1 \\
(1.46-1.85)\end{array}$ & $\begin{array}{l}1.71 \pm 0.09 \\
(1.56-1.85)\end{array}$ & $\begin{array}{l}1.61 \pm 0.07 \\
(1.53-1.72)\end{array}$ & $\begin{array}{l}1.61 \pm 0.13 \\
(1.46-1.76)\end{array}$ \\
\hline
\end{tabular}

Table 2: Patient characteristics in general and by a group.

The intraepidermal nerve fibers density (INFD/mm) is similar between the group with family history $6.8 \pm 2.1(3.5-10.1)$ and in the diabetics $6.3 \pm 2.9(3.5-7.05)$ while the control group reported a density of $10 \pm 1.2$
$(8.2-10.1)$ with a $p=0.01$ between the three groups. Figure 1 . Figures 2 , 3 , and 4 show photographs of the epidermis of the three study groups. 


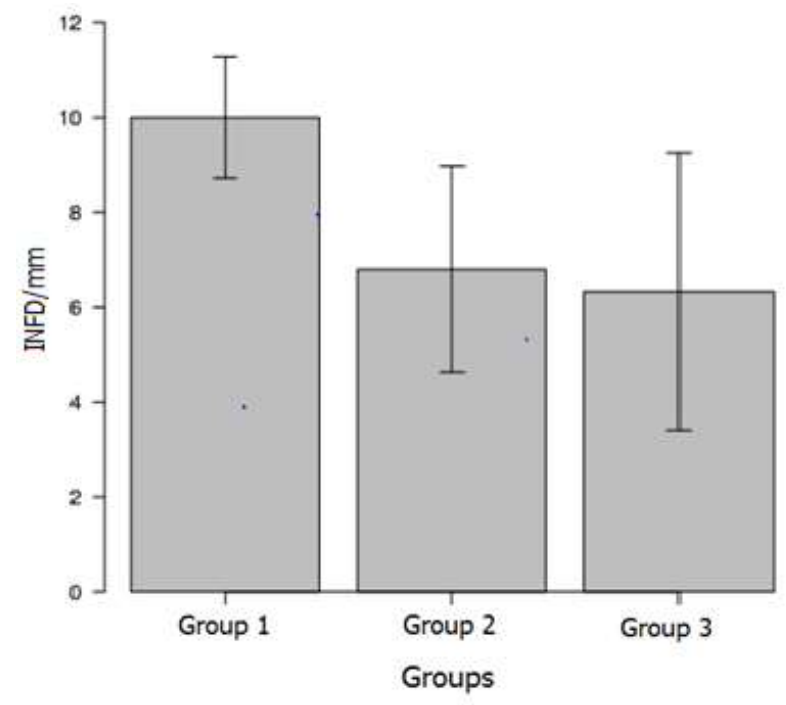

Figure 1: Intraepidermal nerve fiber density in groups 1,2, and 3.

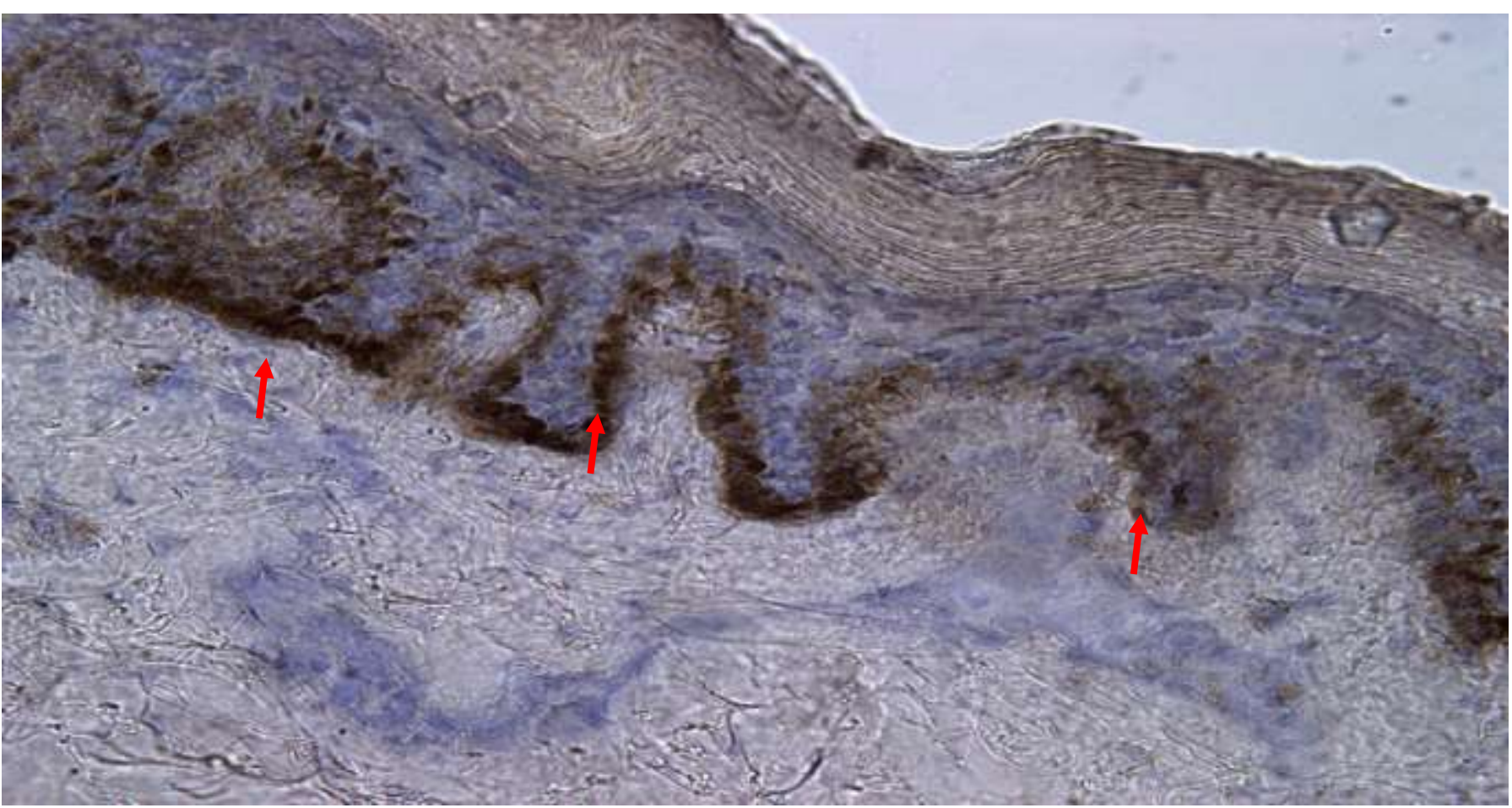

Figure 2: Skin biopsy healthy subject, epidermis without alterations, and nerve fibers stained with PGP 9.5 vertically reach and cross the dermoepidermal junction (red arrows), up to the stratum spinosum. 


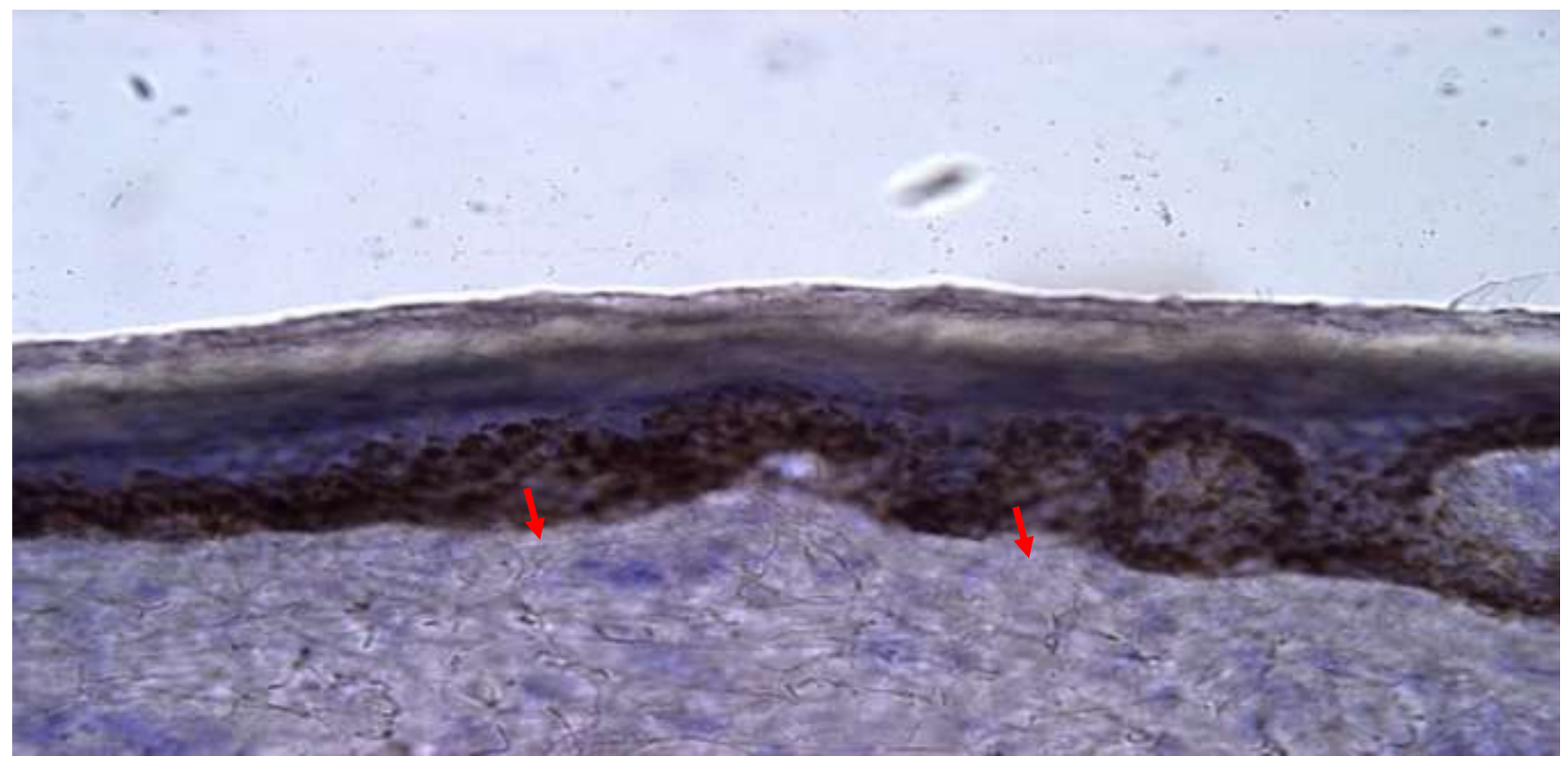

Figure 3: Skin biopsy subject with diabetic family history. The thinned epidermis, horizontalization of the nerve fibers (arrows) with thinning of these, and less penetration of the dermo-epidermal junction are observed.

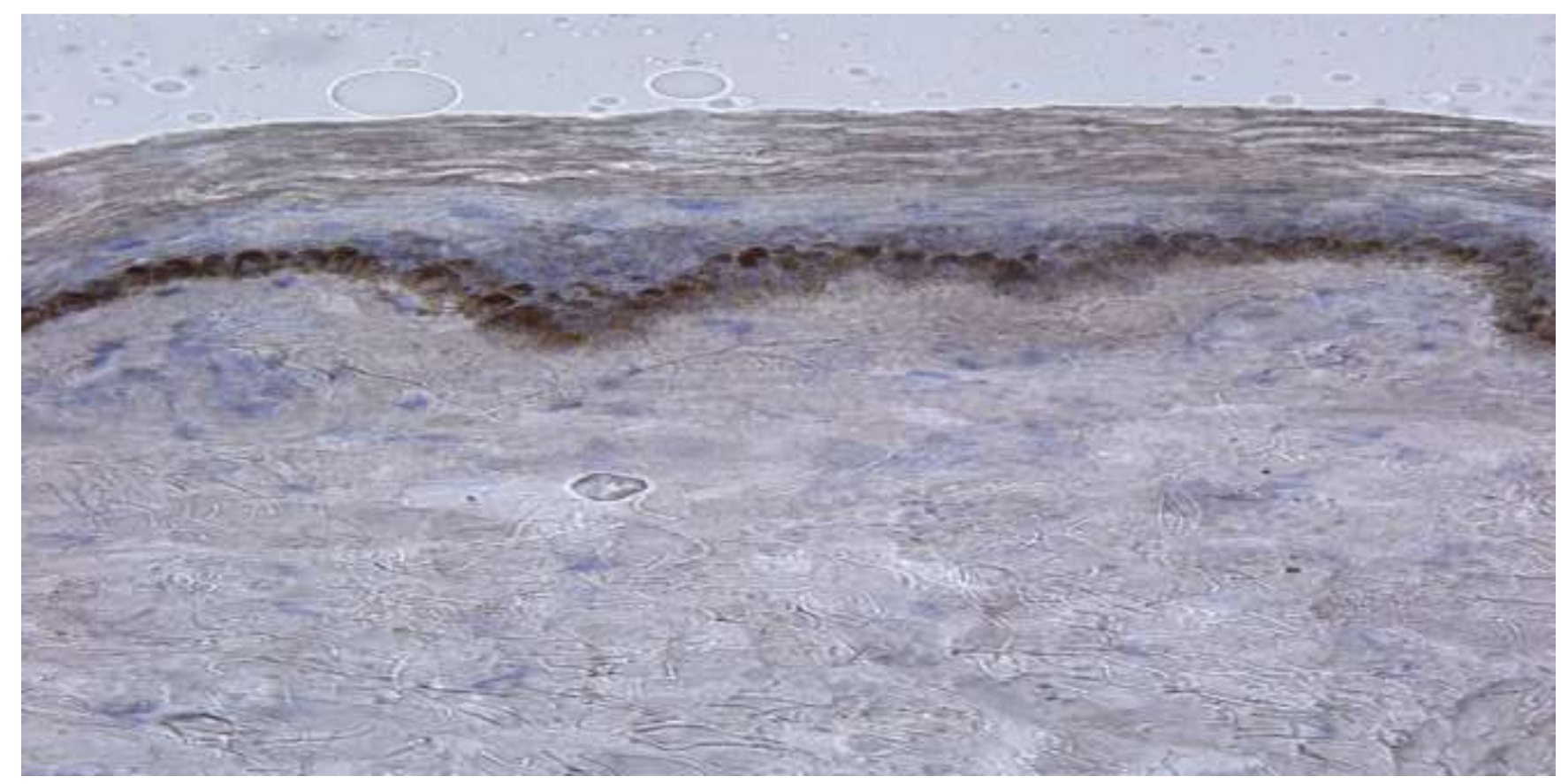

Figure 4: Skin biopsy subject with DM2. The epidermis is atrophic, with depopulation of nerve fibers, and practically none of these reach the dermoepidermal junction, in addition to total horizontalization of the same in the dermis.

Body mass index (BMI) was 28.8 (22.7 - 40.2) for patients with DM2 compared to controls 24.8 (23.35 - 26.9) and subjects diabetic family history 24.9 (19.2 - 36.7) (Figure 5). 

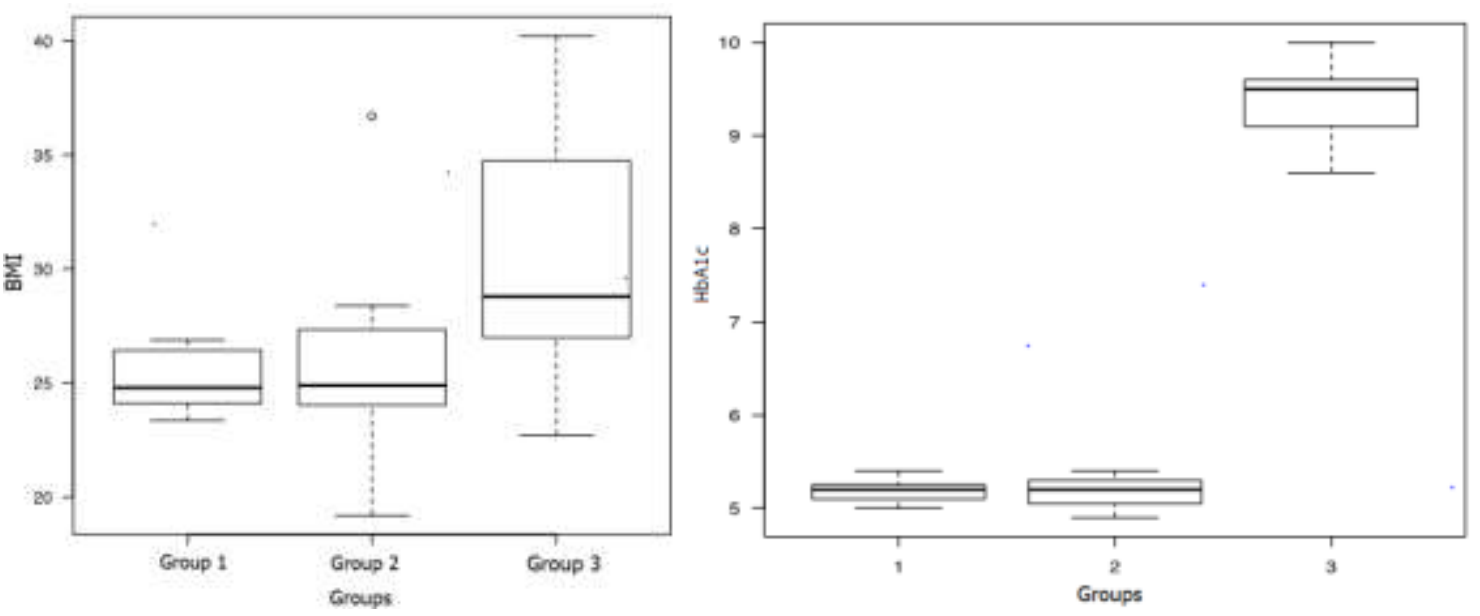

Figure 5a: Body mass index by study group. Figure 5b: Glycosylated hemoglobin values by study group

Glycosylated hemoglobin (HbA1c) was 5.2 (5-5.4) for group 1, 5.2 (4.9-5.4) in group 2 and 9.5 (6.8-10) in group 3 with a p=0.001. (Figure 5b). The decrease in INFD showed a tendency to decrease with increasing age and BMI with a relationship coefficient for age of $\mathrm{r}=-0.342,95 \% \mathrm{CI}(-0.67$ - 0.106), $\mathrm{p}=0.129$; and for BMI of $\mathrm{r}=-0.36,95 \% \mathrm{CI}(-0.685-0.0847), \mathrm{p}=0.109$. (Figures 7 and 8$)$.
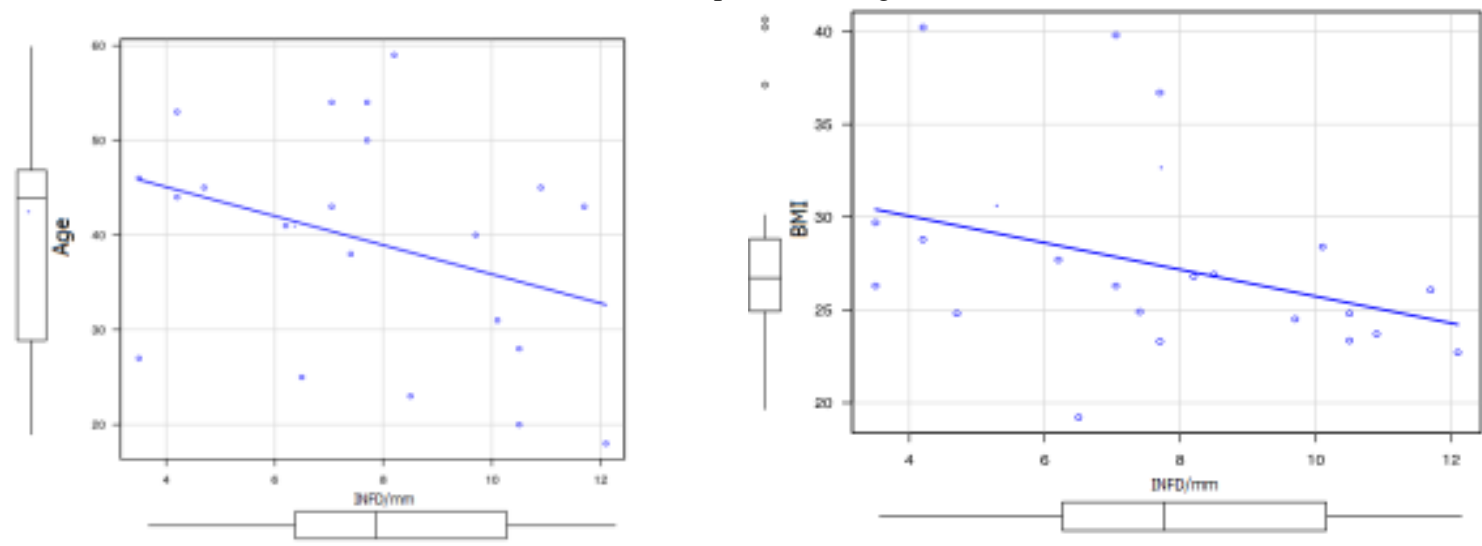

Figure 6a: Correlation between the density of intraepidermal nerve fibers and age in years. Figure 6b: Correlation between intraepidermal nerve fiber density and body mass index.

No direct correlation was found between HbA1c levels and INFD/mm with $r=-0.375,95 \%$ CI $-0.694-0.068$, $p=0.0942$. (Figure 7).

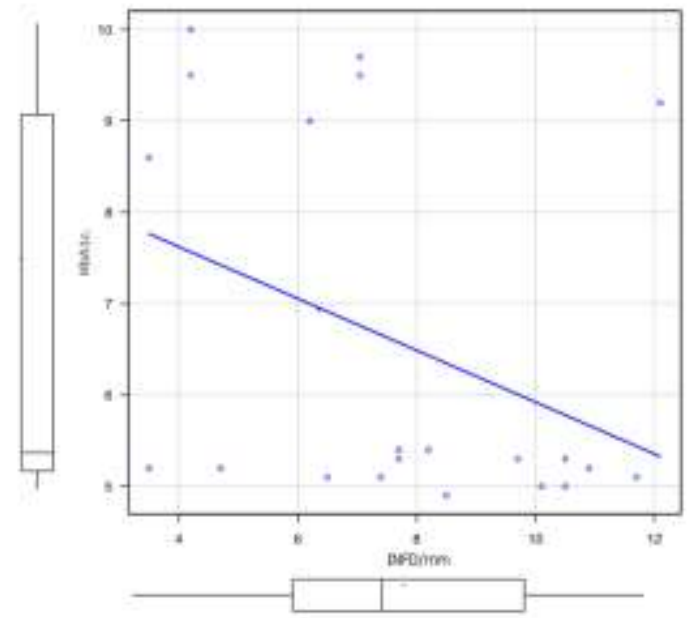

Figure 7: Correlation between the density of intraepidermal nerve fibers and HbAlc. 
In the multivariate linear regression analysis to evaluate the factors associated with the variation of INFD/mm, the initial model included the group, $\mathrm{HbA1c}$, and BMI, leaving in the final model only the group, in the relatives of patients with diabetes, the variation was $-2.7 / \mathrm{mm}(95 \% \mathrm{CI}-$ $4.8--0.58)$ and in the diabetic group $-3.14 / \mathrm{mm}$ (95\% CI $-5.2--1.0$ ) concerning healthy patients, this model explains $38.4 \%$ of the variation ( $p$ $=0.01)$.

\section{Discussion}

The presence of DM2 in any first-degree relative increases the subject's lifetime risk of presenting DN in 2 to 3 more times. If both parents raise DM2, the risk increases 5 to 6 more times $[19,20]$ The fact that the group with diabetic family history and diabetes present a similar INFD/mm is exciting since it can be inferred that this population is at imminent risk not only of giving DM2; but one of the complications that have the most significant socioeconomic impact on the people.

As described by Gallo et al [21], Mexicans show a greater tendency to develop diabetes; our study is the first to evaluate the INFD/mm in Mexicans, suggesting that this is also a population with a high risk of developing diabetic neuropathy. These results indicate that alterations in INFD/mm begin even before a diagnosis of prediabetes or diabetes is established if the subject already has a genetic load for DM2, being the first study worldwide to describe these findings.

Diabetic neuropathy causes disability due to pain, loss of sensation, gait instability, injuries secondary to falls, ulceration, and amputation of the foot.

Although there are no specific medical treatments and effective glycemic control, overweight control and exercise could change the future lives of those who do not know they have diabetes but have all the potential to be so and certainly to suffer from neuropathy be presented in a previous form.

Skin biopsy for detecting intraepidermal nerve fibers is a minimally invasive procedure that can be of great utility as a screening method in subjects at high risk of developing DM2, perhaps the most pertinent candidates for those subjects with high BMI and family history. As described in the literature, our study shows an inversely proportional relationship between increasing BMI and INFD/mm. HbA1c does not seem to directly connect subjects with a family history of DM2 for the decrease in INFD/mm, so perhaps a prevention strategy in these subjects is an adequate exercise plan.

We could not determine at what age and what other risk factors would be essential to choose the test candidates. Therefore, obtaining a larger sample would enrich the possible parameters to generate a scale or score that would allow us to establish which subjects would be convenient to examine.

Objectively indicating that the subject has morphometric alterations in nerve fibers' density (even without a serological diagnosis of prediabetes or diabetes) can open the door to new strategies. The first contact physician could apply those strategies for the prevention and awareness of the population and then reduce the complications and costs caused by these pathologies.

This study's main limitation is the small sample population; even though the results show a clear tendency to decrease INFD/mm in subjects with diabetic family history it is necessary to have a larger sample to increase the statistical performance of the test.
It is necessary to generate a strategy to establish which subjects are ideal for testing and obtaining data to help the clinician.

The study must continue recruiting participants in the coming years to establish this procedure as one of the screening tools in the arduous work of health education and prevention.

\section{Conclusions}

Intraepidermal nerve fiber density is decreased in subjects with a family history of diabetes in comparison with healthy subjects, leading to the possibility to use de skin biopsy as an early biomarker to detect neuropathy. The Mexicans as a population present a higher risk to develop DM2 and diabetic neuropathy, according to the results of this study. Agreeing to previous literature higher BMI and older age are significant risk factors for the decrease of INFD/mm. Glycosylated hemoglobin does not correlate directly with fiber density. Height, weight, and gender in this study were not associated with a decrease in INFD/mm. To our knowledge this is the first study realized in the Mexican population, we propose to continue studying this parameter trying to establish an early biomarker of diabetes o neuropathy.

\section{References}

1. Vinik AI. Diabetic sensory and motor neuropathy. N Engl J Med. 2016;374(15):1455-1464.

2. Pop-Busui R, Boulton AJ, Feldman EL, Bril V, Freeman R, Malik RA, et al. Diabetic neuropathy: a position statement by the American Diabetes Association. Diabetes Care. 2017;40(1):136-154.

3. Perkins BA, Olaleye D, Zinman B, Bril V. Simple screening tests for peripheral neuropathy in the diabetes clinic. Diabetes Care. 2001;24(2):250-256.

4. Ziegler D, Papanas N, Vinik AI, Shaw JE. Epidemiology of polyneuropathy in diabetes and prediabetes. Handb Clin Neurol. 2014;126:3-22.

5. Sommer C, Lauria G. Skin biopsy in the management of peripheral neuropathy. Lancet Neurol. 2007;6(7):632-642.

6. Lauria G, Lombardi R, Camozzi F, Devigili G. Skin biopsy for the diagnosis of peripheral neuropathy. Histopathology. 2009;54(3):273-285.

7. Lauria G, Lombardi R. Skin biopsy: a new tool for diagnosing peripheral neuropathy. Bmj. 2007;334(7604):1159-1162.

8. Pittenger GL, Ray M, Burcus NI, McNulty P, Basta B, Vinik AI. Intraepidermal nerve fibers are indicators of small-fiber neuropathy in both diabetic and nondiabetic patients. Diabetes Care. 2004;27(8):1974-1979.

9. Kennedy WR, Wendelschafer-Crabb G, Johnson T. Quantitation of epidermal nerves in diabetic neuropathy. Neurology. 1996;47(4):1042-1048.

10. Levy D, Karanth S, Springall D, Polak J. Depletion of cutaneous nerves and neuropeptides in diabetes mellitus: an immunocytochemical study. Diabetologia. 1989;32(7):427-433.

11. Levy D, Terenghi G, Gu X-H, Abraham R, Springall D, Polak J. Immunohistochemical measurements of nerves and neuropeptides in diabetic skin: relationship to tests of neurological function. Diabetologia. 1992;35(9):889-897.

12. Lauria G, Hsieh ST, Johansson O, Kennedy WR, Leger JM, Mellgren SI, et al. European Federation of Neurological Societies/Peripheral Nerve Society Guideline on the use of skin biopsy in the diagnosis of small fiber neuropathy. Report of a joint task force of the European Fe-deration of Neurological 
Societies and the Peripheral Nerve Society. Eur J Neurol. 2010;17(7):903-e949.

13. Lauria G, Bakkers M, Schmitz C, Lombardi R, Penza P, Devigili $\mathrm{G}$, et al. Intraepidermal nerve fiber density at the distal leg: a worldwide normative reference study. J Peripher Nerv Syst. 2010;15(3):202-207.

14. Karlsson P, Haroutounian S, Polydefkis M, Nyengaard JR, Jensen TS. Structural and functional characterization of nerve fibres in polyneuropathy and healthy subjects. Scand J Pain. 2016;10:28-35.

15. Narayanaswamy H, Facer P, Misra V, Timmers M, Byttebier G, Meert T, et al. A longitudinal study of sensory biomarkers of progression in patients with diabetic peripheral neuropathy using skin biopsies. J Clin Neurosci. 2012;19(11):1490-1496.

16. Divisova S, Vlckova E, Srotova I, Kincová S, Skorna M, Dusek $\mathrm{L}$, et al. Intraepidermal nerve-fibre density as a biomarker of the course of neuropathy in patients with Type 2 diabetes mellitus. Diabet Med. 2016;33(5):650-654.
17. Browne RH. On the use of a pilot sample for sample size determination. Stat Med. 1995;14(17):1933-1940.

18. Team RC. R: A language and environment for statistical computing. R Foundation for Statistical Computing,[on line]. Vienna, Austria. 2014;

19. Meigs JB, Cupples LA, Wilson P. Parental transmission of type 2 diabetes: the Framingham Offspring Study. Diabetes. 2000;49(12):2201-2207.

20. InterAct Consortium robert. scott@ mrc-epid. cam. ac. uk. The link between family history and risk of type 2 diabetes is not explained by anthropometric, lifestyle or genetic risk factors: the EPIC-InterAct study. Diabetologia. 2013;56:60-69.

21. Gallo LC, Penedo FJ, Carnethon M, Isasi C, Sotres-Alvarez D, Malcarne VL, et al. The Hispanic community health study/study of Latinos sociocultural ancillary study: Sample, design, and procedures. Ethn Dis. 2014;24(1):77.

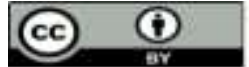

This work is licensed under Creative Commons Attribution 4.0 License

To Submit Your Article Click Here: Submit Manuscript

DOI: $10.31579 / 2578-8868 / 169$
Ready to submit your research? Choose Auctores and benefit from:

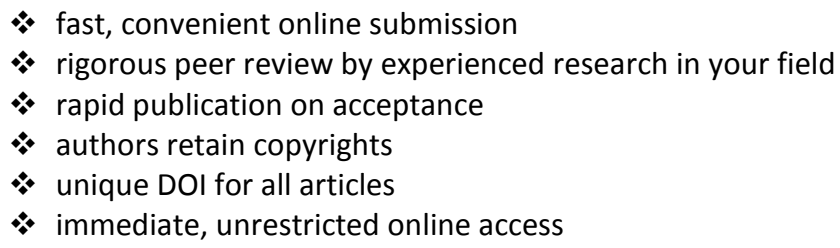

At Auctores, research is always in progress.

Learn more www.auctoresonline.org/journals/neuroscience-andneurological-surgery 